\title{
Sharing the responsibility of prostate cancer risk reduction
}

Laurence Klotz, MD, FRCSC; Yves Fradet, MD, FRCSC; Larry Goldenberg, $O B C, M D, F R C S C, F A C S$; Simon Tanguay, MD, FRCSC

Co-editors, CUAJ supplement "Understanding Prostate Cancer Risk Reduction: 2009 Update"
$\mathrm{D}$ espite improvements in the detection and management of prostate cancer in recent years, the clinical and financial burden of this disease continues to grow. Prostate cancer remains the most frequently diagnosed cancer in men and a leading cause of cancer death worldwide, yet there is still ongoing uncertainty surrounding the value of early screening. Intuitively, earlier detection should result in earlier and more effective treatment of prostate cancer; however, this has yet to be supported by the evidence, emphasized by the recent conflicting reports on screening from the Prostate, Lung, Colorectal and Ovarian (PLCO) and European trials. Still, as with any cancer, a diagnosis of prostate cancer carries a significant burden, and exploring the cost-benefit of risk reduction remains worthwhile.

With primary care physicians assuming an increasingly greater role in the management of benign prostatic hyperplasia (BPH) and lower urinary tract symptoms in general, urologists and primary care physicians are moving toward a more cooperative approach to the diagnosis and management of these conditions. This cooperation results in greater access to care and improved outcomes, as well as a reduced need for unnecessary surgical consultations.

This supplement to the Canadian Urological Association Journal grew out of a series of meetings of the Prostate Cancer Collaborative Network (PCCN) - a multidisciplinary group of Canadian physicians dedicated to advancing the management of prostate cancer in Canada. Supported by an unrestricted educational grant from GlaxoSmithKline, the PCCN convened to explore new approaches to prostate cancer risk reduction.

Along with their colleagues in the PCCN, Dr. Simon Tanguay discusses the shared roles of urology and primary care in the diagnosis and management of $\mathrm{BPH}$, Dr. Yves Fradet provides a Canadian perspective on the burden of prostate disease, Dr. Larry Goldenberg reviews the role of 5-alpha reductase in prostate disease and Dr. Laurence Klotz addresses recent issues with Gleason grading.

We hope this series of articles provides the reader with a comprehensive review of the current issues facing urologists, oncologists, radiation oncologists and primary care physicians involved in the management of prostate disease. 